\title{
Comparative study of surgical outcomes between early decompressive craniectomy with clipping and coil embolization followed by decompressive craniectomy
}

\author{
Je Min Choi, Ik Seong Park \\ Department of Neurosurgery, Bucheon St. Mary's Hospital, College of Medicine, The Catholic University of Korea, Bucheon, Korea
}

Objective: Poor-grade aneurysmal subarachnoid hemorrhage (aSAH) has high morbidity and mortality, even when emergency treatment such as decompressive craniectomy (DC), coil embolization, or clipping is performed. The best treatment for acute aSAH, especially in poor-grade aSAH patients, has not been determined. The purpose of this study was to evaluate treatment methods in these patients in order to suggest the best treatment method.

Methods: We compared 130 patients with poor-grade aSAH who underwent DC with clipping or coiling (clipping, 102 patients; coiling, 28 patients). We compared functional outcome, mortality, and the time interval between admission and DC surgery between the clipping and coiling groups.

Results: There was a significant difference in functional outcomes (modified Rankin score [mRS]) between the clipping and coiling groups. The mean $m R S$ at discharge in the clipping and coil groups was 4.824 and 5.214 , respectively $(P=0.049)$. The time interval until DC surgery was also significantly different (161 and 481 minutes in the clipping and coiling groups, respectively; $P=0.003$ ). No significant difference was found in mortality between the 2 groups $(P=0.301)$.

Conclusion: DC might be helpful for severe brain edema and intracranial pressure control. This procedure was more effective when performed with clipping than with coil embolization. DC with clipping showed better functional outcomes, lower mortality, and more favorable outcomes than DC with coil embolization. This demonstrates that aggressive surgical treatment can be helpful for poorgrade aSAH patients.

Keywords: Subarachnoid hemorrhage; Poor grade; Clipping; Coil embolization

\section{Introduction}

Aneurysmal subarachnoid hemorrhage (aSAH) is caused by rupture of an intracranial aneurysm and has high morbidity and mortality. Cases of poor grade aSAH have even higher morbidity and mortality [1-3]. Poor grade aSAH refers to modified Fisher grade 3 or higher and Hunt and Hess (H-H) grade 4 or higher aSAH. In

Received: September 8, 2021

Revised: October 8, 2021

Accepted: October 9, 2021

Corresponding Author: Ik Seong Park, MD, PhD

Department of Neurosurgery, Bucheon St. Mary's Hospital, College of Medicine, The Catholic University of Korea, 327 Sosa-ro, Wonmi-gu, Bucheon 14647, Korea

Tel: +82-32-340-7212; E-mail: ispark@catholic.ac.kr such patients, the possibility of neurological recovery is especially low due to the initial brain injury or increased intracranial pressure (IICP). Therefore, immediate control of IICP in the acute phase is very important [4]. Surgical treatment includes external ventricular drain (EVD), hematoma evacuation during clipping, and craniectomy. Although the utilization of decompressive craniectomy (DC) in aSAH patients is controversial, some highly regarded specialized institutions have demonstrated that $\mathrm{DC}$ decreases mortality and morbidity [5-14].

Traditionally, simultaneous craniectomy with aneurysmal neck clipping has been used in poor grade ruptured cerebral aneurysm patients. However, when brain swelling is severe, the process of dissecting the subarachnoid space for ligation of the cerebral aneurysm and securing the parent artery and aneurysm can cause further brain damage. Recently, endovascular coil embolization has 
been developed, and its use has been increasing. This procedure attempts to control IICP by first preventing re-bleeding using a coil and then performing DC. Although there is no additional brain damage around the ruptured aneurysm during coil embolization, there is a risk of further brain damage in severe IICP cases due to brain edema during this procedure. DC can be conducted first, followed by coil embolization, but this method carries the risk of re-bleeding. The authors conducted this study to test the methods of treatment so that we might suggest a best treatment method for poor-grade patients. Our test was conducted by examining how the prognosis after surgery differed between patients who received simultaneous clipping and DC and patients who first received coil embolization and then DC.

\section{Material and Method}

\section{Patient inclusion criteria}

We included patients who visited our hospital with poor grade aSAH (World Federation of Neurological Surgeons or $\mathrm{H}-\mathrm{H}$ grade 3 or more) and underwent DC between April 2004 and January 2021. We excluded patients who received DC due to re-rupture during or after surgery or DC due to delayed cerebral ischemia as these patients were low grade aSAH at the time of admission. Cases in which minimal craniectomy was performed instead of general decompression (size of removed bone flap less than $12 \times 15 \mathrm{~cm}$ ) were also excluded (17 patients).

The number of patients who received clip and DC was 102, and the number of patients who received coil and DC was 28 . We used brain computed tomography $(\mathrm{CT})$ and brain $\mathrm{CT}$ angiography to diagnose aSAH. The treatment plan decision (clip or coil) was made by the officiating, well-experienced vascular neurosurgeon(s). The decision to perform $\mathrm{DC}$ was based on factors such as brain edema, herniation, presence of intracerebral hematoma (ICH), and intra-operative brain swelling $[7,10,13]$.

\section{Surgery and post-surgical management}

Coil embolization performance was preceded by bi-plane digital subtraction angiography (DSA) under general anesthesia. DC was performed after coil embolization, and decompression measures to remove bone flaps larger than $12 \times 15 \mathrm{~cm}$ were performed. Hematoma evacuation was performed according to the decision of the neurosurgeon, and 26 of 28 patients received both EVD insertion and DC. Clipping of the aneurysm and DC were performed simultaneously, and this also included removal of large bone flaps $(12 \times 15 \mathrm{~cm}$ or more) .

After surgery, all patients were monitored in the intensive care unit. We designed our own aSAH treatment protocol in accor- dance with best medical treatment guidelines. This included treatment methods to prevent worsening of complications that can occur after aneurysm rupture $[2,15]$. Postoperative follow-up was performed through brain $\mathrm{CT}$, cerebral $\mathrm{CT}$ angiography, magnetic resonance imaging, magnetic resonance angiography, and DSA. When necessary, chemical angioplasty was performed for vasospasm. EVD changes were performed every 1 to 2 weeks to prevent infection.

\section{Outcome measurements and statistical analysis}

We recorded $\mathrm{H}-\mathrm{H}$ grade and modified Fisher grade of the aSAH patients, and functional outcomes of the patients were recorded in accordance with the modified Rankin score (mRS). Our goal was to test whether there was any difference in functional outcome of patients in whom coil or clip was performed. We assessed mRS at the time of admission and at the time of discharge, comparing the difference in $\mathrm{mRS}$ at these times. Patient characteristics included age, sex, length of hospital stay, location, and $\mathrm{H}-\mathrm{H}$ and modified Fisher grades. Also, the time interval between hospital arrival and time of DC surgery was ascertained. Statistical analyses included performance of unpaired t-test, chi-square test, and Fisher exact test using IBM SPSS software ver. 20.0 (IBM Corp., Armonk, NY, USA). We calculated the $95 \%$ confidence interval (CI), and $\mathrm{P}<0.05$ was considered significant.

\section{Results}

Patient characteristics and demographics are summarized in Table 1. In the clip and coil groups, mean age was $53.07 \pm 12.48$ years and $55.89 \pm 10.57$ years, respectively. The difference was not statistically significant ( $95 \% \mathrm{CI},-7.54$ to $1.90 ; \mathrm{P}=0.235$ ). Forty-two males and 60 females were in the clip group; 12 males and 16 females were in the coil group. There was no significant difference between the 2 groups in sex distribution $(P=0.520)$. However, there was a significant difference in length of hospital stay between the 2 groups. Mean hospital stay for the clip group was 45 days, while that for coil patients was 30 days (mean \pm standard deviation [SD] value of clipping vs. coiling groups $45.66 \pm 39.54$ and $30.04 \pm 23.55$, respectively; $\mathrm{P}=0.010$ ). For the location of the aneurysm, in clip patients, middle cerebral artery (MCA) aneurysm was the most common with 61 (59.8\%), followed by 25 (24.5\%) anterior cerebral artery (ACA) aneurysms and $16(15.7 \%)$ internal carotid artery (ICA) aneurysms. In the coil group, there were 15 (53.6\%) MCA aneurysms, 8 (28.6\%) ACA aneurysms, and 5 (17.9\%) ICA aneurysms. There was no significant difference between the groups $(\mathrm{P}=0.833)$. There were no significant differences between the clip and coil groups for $\mathrm{H}-\mathrm{H}$ 
Je Min Choi, et al.

Table 1. Patient characteristics

\begin{tabular}{|c|c|c|c|c|}
\hline Characteristic & Clip $(n=102)$ & Coil $(n=28)$ & $95 \% \mathrm{Cl}$ & P-value \\
\hline Age (yr) & $53.07 \pm 12.48$ & $55.89 \pm 10.57$ & -7.54 to 1.90 & 0.235 \\
\hline Sex (male:female) & $42: 60$ & $12: 16$ & & 0.520 \\
\hline Hospital stay (day) & $45.66 \pm 39.54$ & $30.04 \pm 23.55$ & $5.74-32.01$ & 0.010 \\
\hline Location & & & & 0.833 \\
\hline Anterior cerebral artery & 25 & 8 & & \\
\hline Middle cerebral artery & 61 & 15 & & \\
\hline Internal carotid artery & 16 & 5 & & \\
\hline Hunt and Hess grade & & & & 0.413 \\
\hline 4 & 83 & 24 & & \\
\hline 5 & 2 & 4 & & \\
\hline Modified Fisher grade & & & & 0.523 \\
\hline 2 & 1 & & & \\
\hline 3 & 0 & 9 & & \\
\hline 4 & 61 & 19 & & \\
\hline Time interval (min) & $161.529 \pm 169.21$ & $481.821 \pm 512.44$ & $119.17-521.41$ & 0.003 \\
\hline
\end{tabular}

Values are presented as mean \pm standard deviation. The time interval indicates the duration from hospital arrival to decompressive craniectomy surgery. The 95\% confidence interval (Cl) was calculated using the t-test.

Table 2. Functional outcomes (modified Rankin score at discharge) in the clipping and coil groups

\begin{tabular}{|c|c|c|c|c|c|c|c|}
\hline \multirow{2}{*}{ Group } & \multicolumn{7}{|c|}{ Modified Rankin score } \\
\hline & 1 & 2 & 3 & 4 & 5 & 6 & Total \\
\hline \multicolumn{8}{|l|}{ Clipping } \\
\hline Admission & 0 & 0 & 0 & $1(1.0)$ & 101 (99.0) & 0 & 102 \\
\hline Discharge & $1(1.0)$ & $4(3.9)$ & $10(9.8)$ & 15 (14.7) & 39 (38.2) & $33(32.4)$ & 102 \\
\hline \multicolumn{8}{|l|}{ Coil } \\
\hline Admission & 0 & 0 & $1(3.6)$ & $1(3.6)$ & 26 (92.9) & 0 & 28 \\
\hline Discharge & 0 & 0 & $1(3.6)$ & $4(14.3)$ & 11 (39.3) & $12(42.9)$ & 28 \\
\hline
\end{tabular}

Values are presented as number (\%).

or Fisher grade $(\mathrm{H}-\mathrm{H}, \mathrm{P}=0.413$; Fisher grade, $\mathrm{P}=0.523)$. The time interval difference between hospital arrival and DC surgery was significant. The mean time interval was 161 minutes in the clip group and 481 minutes in the coil group (mean \pm SD value clipping vs. coiling groups $161.529 \pm 169.21$ and $481.821 \pm 512.44$, $\mathrm{P}=0.003$ ). This result suggests that the timing of DC surgery might be an important prognostic factor for poor-grade aSAH patients.

We found no significant difference in admission mRS, with an average value of 4.990 in the clip group and 4.890 in the coil group $(\mathrm{P}=0.117)$ (Table 2$)$. However, discharge $\mathrm{mRS}$ was significantly different between the clip and coil groups $(\mathrm{P}=0.049)$ (Table 3$)$. The average discharge $\mathrm{mRS}$ of the clip group was 4.824 , and $\mathrm{mRS}$ 5 was the most common assessment (39 patients). The discharge $\mathrm{mRS}$ of the coil group averaged 5.214, and mRS 6 was the most common assessment (12 patients). Mortality difference between the 2 groups was not significant $(\mathrm{P}=0.301)$. Thirty-three deaths occurred in the clip group, and 12 deaths occurred in the coil group. The number of patients discharged with good functional
Table 3. Modified Rankin score (mRS) and mortality differences between the clipping and coil groups

\begin{tabular}{lccc}
\hline mRS & Clipping group & Coil group & P-value \\
\hline Admission & 4.990 & 4.890 & 0.117 \\
Discharge & 4.824 & 5.214 & 0.049 \\
Mortality (\%) & $33(32.4)$ & $12(42.9)$ & 0.301 \\
\hline
\end{tabular}

outcome (mRS $0-3)$ was 15 in the clip group and 1 in the coil group, and the difference between the 2 groups was not significant $(\mathrm{P}=0.075)$.

\section{Case presentation}

\section{Case 1. Clipping}

A 63-year-old male patient came to emergency department of The Catholic University of Korea, Bucheon St. Mary's Hospital, he complained of sudden bursting headache and progressed to mental deterioration brain $\mathrm{CT}$ revealed thick $\mathrm{SAH}$ and $\mathrm{ICH}$ due to ruptured MCA aneurysm in right frontal lobee (Fig. 1). Initial 

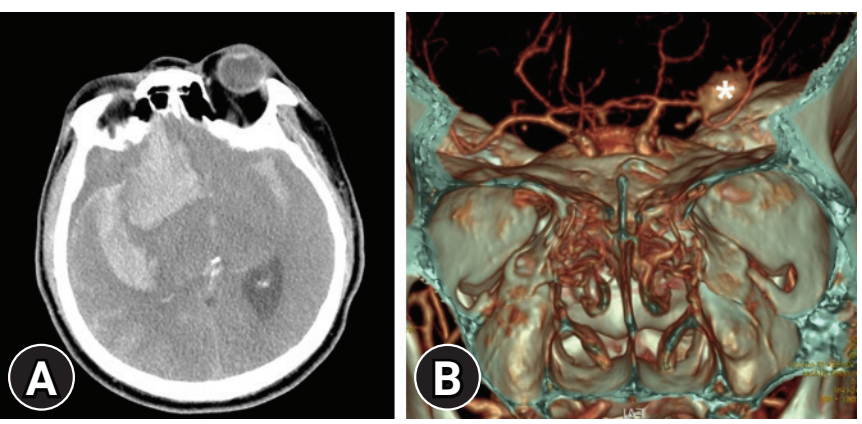

Fig. 1. Preoperative images. (A) Brain computed tomography (CT) revealed a thick subarachnoid hemorrhage on the right sylvian fissure and intracerebral hemorrhage in the right frontal lobe. (B) Surface rendering image of CT angiography showed a saccular aneurysm $(*)$ on the right middle cerebral artery bifurcation.

clinical grade was H-H grade 5 and Radiologically Fisher grade 4. He underwent emergency DC with hematoma removal and direct aneurysmal neck clipping (Fig. 2). The patient gradually awoke and became to obey command at 1 months of operation so cranioplasty with autologous bone was performed (Fig. 3). His final status at discharge was mRS 3 .

\section{Case 2. Coiling}

A 70-year-old female patient came to emergency department with stuporous mentality. Initially brain CT showed thick SAH due to ruptured MCA aneurysm on left hemisphere (Fig. 4). Clinical grade was $\mathrm{H}-\mathrm{H}$ grade 4 , and radiologically Fisher group III. She underwent diagnostic DSA and coil embolization of aneurysm right away, and then was followed by DC. During postoperative management, brain swelling had progressed eventually, finally expired 4 days after operation (Fig. 5). Written informed consent was obtained for publication of this case report and accompanying images.

\section{Discussion}

Poor-grade aSAHs generally have high morbidity and mortality. One of the most important factors associated with poor outcome after rupture of a cerebral aneurysm is an initial poor grade [16]. The cause of poor grade after rupture of a cerebral aneurysm is direct brain tissue damage due to bleeding or decreased brain function due to IICP. Brain tissue damage has no treatment and serious sequelae. However, IICP-induced brain functional decline can be prevented if treatment is started before irreversible brain tissue damage occurs. Since IICP is caused mainly by brain edema, DC is useful as an initial treatment. Since the degree of brain damage in poor-grade patients cannot be assessed accurately in the early stag-
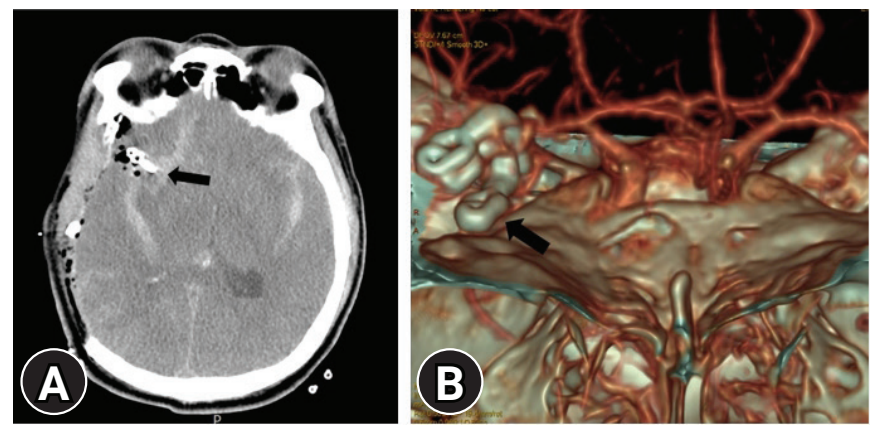

Fig. 2. Postoperative images. (A) Right fronto-temporo-parietal craniectomy with hematoma removal observed on computed tomography (CT) with aneurysmal neck clipping (arrow). (B) Complete aneurysmal neck clipping (arrow) was observed on three-surface rendering of $\mathrm{CT}$.

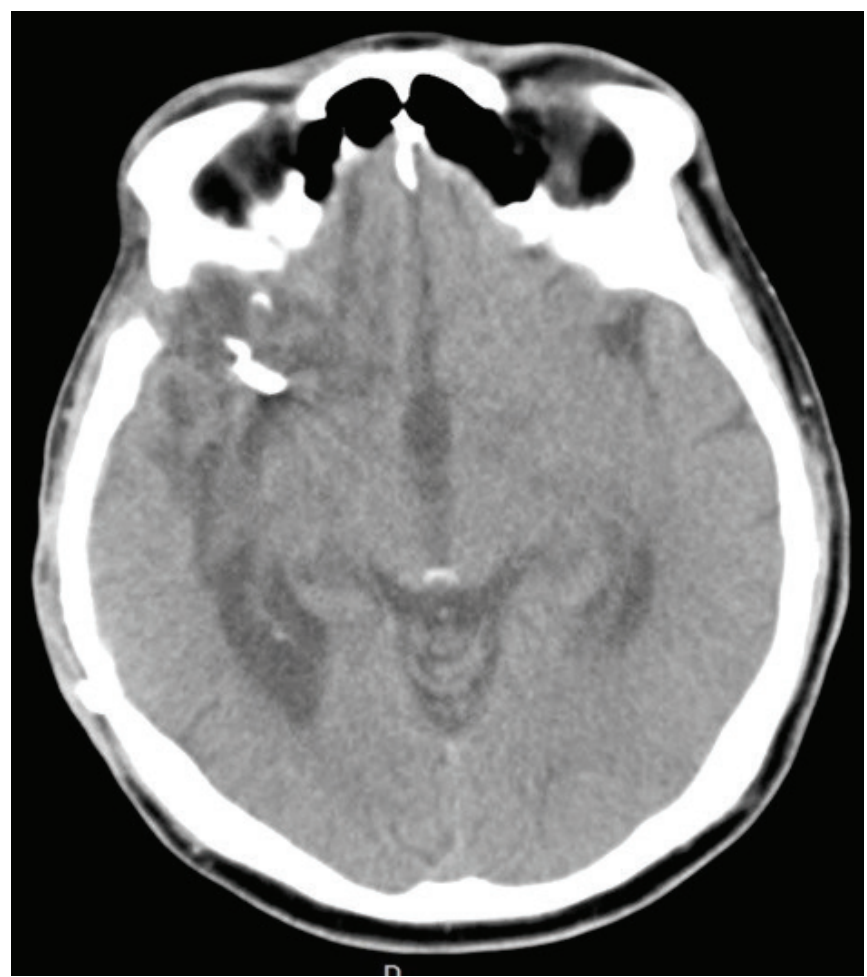

Fig. 3. One-month follow-up image. Post-cranioplasty brain computed tomography showed decreased brain swelling with minimal encephalomalacic changes on the right temporal lobe.

es, distinguishing between patients who will benefit from DC and patients who have already suffered irreversible brain damage is difficult. Therefore, most relevant studies have been retrospective after performance of DC in poor-grade patients. Although the group with DC had lower mortality and morbidity compared to the group without DC, the group with DC showed higher mortality and morbidity compared to the "good" grade group $[5,17]$. Previous studies have shown that DC can reduce mortality without sig- 
nificantly improving functional outcomes [1,4,5,6,17]. Attempting to clip a cerebral aneurysm in a patient with severe brain swelling can cause additional damage to the brain tissue surrounding the cerebral aneurysm. Therefore, direct aneurysmal clipping is more challenging in poor-grade aSAHs than in "good" grade SAHs. In addition, the incidence of delayed cerebral infarction due to vasospasm other than initial brain injury in poor-grade patients contributes to poor outcome. Although controversial, immediate DC and clipping have been accepted as the general treatment for patients with poor-grade aSAH.

As performance of endovascular coil embolization became more popular, better results with this procedure were reported in poorgrade aSAH patients than in the surgical group patients [18]. Later, studies were published on the comparison of DC with clipping treatment and endovascular coiling with DC treatment in poor-
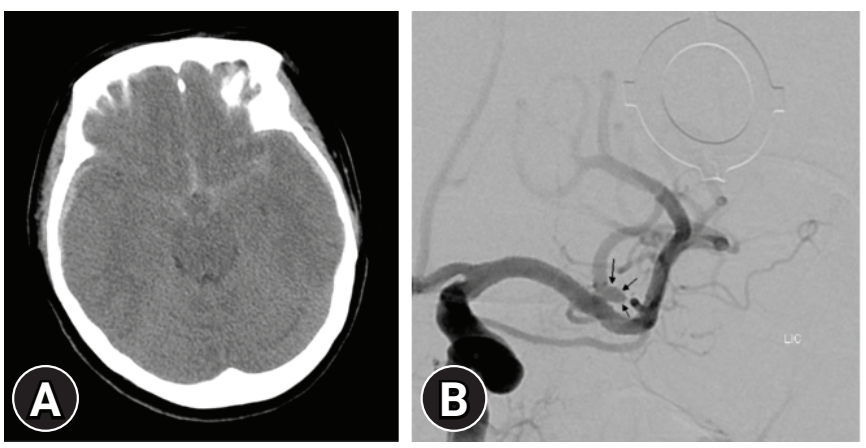

Fig. 4. Preoperative images. (A) Brain computed tomography revealed a subarachnoid hemorrhage with severe brain swelling. (B) Pre-coil embolization digital subtraction angiography image showed an aneurysmal dilatation on the left middle cerebral artery (arrows). grade patients, but the results were inconsistent $[3,9,19]$. The reason that the coil group showed a better prognosis than the surgical group was that brain tissue damage did not occur in the coil group during cerebral aneurysm embolization. However, the high mortality rate compared with the surgery group was due to additional brain damage that occurs during the delay before DC. Hwang et al. [9] tried to prevent re-bleeding in poor-grade patients first by coil embolization and then by DC to resolve IICP. To conduct DC as quickly as possible, coil embolization was performed under sedation rather than under general anesthesia. In this study, not only the outcomes of the surgery group and the coil group, but also the difference in the interval time until DC between the groups was compared and analysed.

The selection of DC with clipping or DC with coiling in poorgrade patients is important and should be determined based on the degree of brain swelling and the risk of re-bleeding according to aneurysm morphology. It is important to evaluate the priority between treatment to prevent re-bleeding and treatment to control IICP and to predict and determine the degree of additional brain damage that can occur in the course of treatment to prevent re-bleeding. The surgeon should first decide whether to use clipping or coiling and then decide whether to perform DC before or after.

The group in which DC and clipping were performed had lower morbidity than the group in which DC and coiling were performed together. There was no difference in $\mathrm{mRS}$ at the time of admission between the 2 groups, but the functional outcomes (mRS) at discharge were better in the group in which clipping was performed. While mortality was not significantly different between clipping and coiling groups, the clipping group showed a
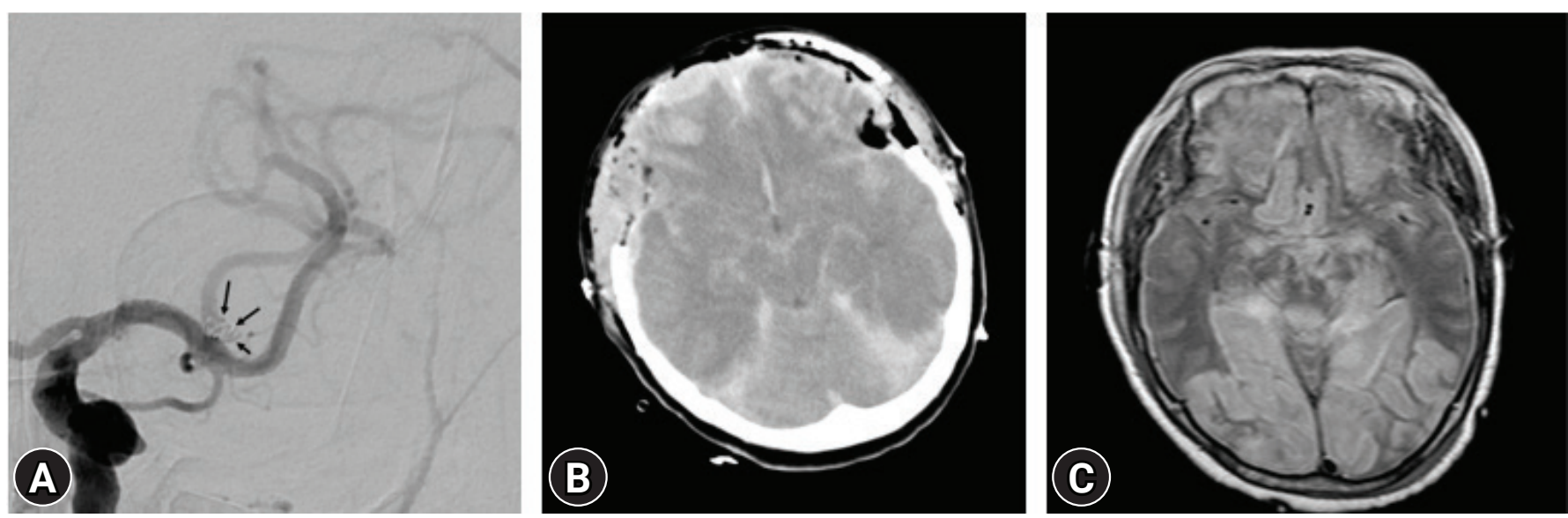

Fig. 5. Postoperative images. (A) Post-coil embolization digital subtraction angiography image revealed complete obliteration of the aneurysmal dilatation by coils (black arrows). (B) Brain computed tomography post-craniectomy (immediately after coil embolization) showed severe brain swelling with diffuse subarachnoid hemorrhage. (C) An ischemic injury of both occipital lobes, suggesting brain herniation, was noticed at postoperative 4 hours on a magnetic resonance fluid attenuated inversion recovery image. 
tendency toward lower mortality than the coil group. Also, a relatively large number of patients discharged with good functional outcome (mRS 0-3) were present in the clipping group. Therefore, in patients presenting with the same neurological deficit, the functional outcome was better at discharge for those in whom DC and clipping were performed simultaneously.

When DC and clipping were performed at the same time, bone flap removal and dura incision were performed more quickly; early decompression resulted in better functional outcomes. SAH evacuation through a trans-sylvian approach and cerebrospinal fluid circulation promotion through a cistern opening can be effective in regulating brain edema. In cases of subdural hematoma and $\mathrm{ICH}$ that have a mass effect with the SAH, the hematoma can be removed at the same time as clipping for decompression.

We divided the patients into 2 groups, those with good functional outcome (mRS 0-3) and those with poor functional outcome (mRS 4-6) and tested to determine the factors causing the difference in effects. The shorter was the time interval between admission and surgery, the better were the Fisher and $\mathrm{H}-\mathrm{H}$ grades, and, therefore, the better was the functional outcome (time interval, $\mathrm{P}=0.001$; Fisher, $\mathrm{P}=0.036 ; \mathrm{H}-\mathrm{H}, \mathrm{P}=0.007$ ). While there was no significant difference in the degree of good functional outcome in the clip and the coil groups, the functional outcome in the clip group showed a better tendency.

\section{Limitations}

The difference in length of hospital stay between groups might have influenced the discharge $\mathrm{mRS}$ value. The longer hospital stay in the clipping group could have allowed for more rehabilitation and recovery and, therefore, a better functional outcome at discharge. Ideally, to adjust for bias created by differences in length of hospital stay, assessing the functional outcome at the same time intervals from admission, such as at 1 month, 6 months, 1 year, would be necessary. However, due to lack of medical records, we did not have the data necessary to perform such an assessment.

This study has the limitations inherent to a single institution, retrospective study with a small number of patients. Selection biases exist, and differences might be due to neurosurgeon decision-making. DC can be easier to perform in conjunction with clipping than with coil embolization; this could contribute to the difference in results. The skill level of the neurosurgeon performing the DC is likely to have an effect on the prognosis, and there might be differences in this skill level between groups.

\section{Conclusion}

To treat poor-grade aSAH, DC might be helpful for cases of severe brain edema and for control of IICP, especially when performed with clipping rather than coil embolization. Also, a short time interval between admission and DC surgery could result in better functional outcomes in poor-grade aSAH cases. Performing DC in conjunction with clipping had more favorable outcomes and demonstrates that aggressive surgical treatment could be helpful for poor-grade aSAH patients.

\section{Conflicts of interest}

No potential conflict of interest relevant to this article was reported.

\section{ORCID}

Je Min Choi, https:/ /orcid.org/0000-0003-1235-9953

Ik Seong Park, https://orcid.org/0000-0002-8187-7204

\section{REFERENCES}

1. Bailes JE, Spetzler RF, Hadley MN, Baldwin HZ. Management morbidity and mortality of poor-grade aneurysm patients. J Neurosurg 1990;72:559-66.

2. Connolly ES Jr, Rabinstein AA, Carhuapoma JR, et al. Guidelines for the management of aneurysmal subarachnoid hemorrhage: a guideline for healthcare professionals from the American Heart Association/American Stroke Association. Stroke 2012;43:1711-37.

3. Zhao B, Rabinstein A, Murad MH, Lanzino G, Panni P, Brinjikji W. Surgical and endovascular treatment of poor-grade aneurysmal subarachnoid hemorrhage: a systematic review and meta-analysis. J Neurosurg Sci 2017;61:403-15.

4. Broderick JP, Brott TG, Duldner JE, Tomsick T, Leach A. Initial and recurrent bleeding are the major causes of death following subarachnoid hemorrhage. Stroke 1994;25:1342-7.

5. Brandecker S, Hadjiathanasiou A, Kern T, Schuss P, Vatter H, Güresir E. Primary decompressive craniectomy in poor-grade aneurysmal subarachnoid hemorrhage: long-term outcome in a single-center study and systematic review of literature. Neurosurg Rev 2021;44:2153-62.

6. Arikan F, Vilalta J, Romero FJ, Porta I, Martínez-Ricarte FR, Sahuquillo J. Primary decompressive craniectomy in patients with aneurysmatic subarachnoid hemorrhage: results of a pilot study in 11 cases. Neurocirugia (Astur) 2010;21:452-60.

7. Buschmann U, Yonekawa Y, Fortunati M, Cesnulis E, Keller E. Decompressive hemicraniectomy in patients with subarachnoid hemorrhage and intractable intracranial hypertension. Acta Neu- 
rochir (Wien) 2007;149:59-65.

8. D’Ambrosio AL, Sughrue ME, Yorgason JG, et al. Decompressive hemicraniectomy for poor-grade aneurysmal subarachnoid hemorrhage patients with associated intracerebral hemorrhage: clinical outcome and quality of life assessment. Neurosurgery 2005;56:12-20.

9. Hwang US, Shin HS, Lee SH, Koh JS. Decompressive surgery in patients with poor-grade aneurysmal subarachnoid hemorrhage: clipping with simultaneous decompression versus coil embolization followed by decompression. J Cerebrovasc Endovasc Neurosurg 2014;16:254-61.

10. Jabbarli R, Oppong MD, Dammann P, et al. Time is brain!: analysis of 245 cases with decompressive craniectomy due to subarachnoid hemorrhage. World Neurosurg 2017;98:689-94.

11. Jussen D, Marticorena S, Sandow N, Vajkoczy P, Horn P. U1tra-early decompressive hemicraniectomy in aneurysmal intracerebral hemorrhage: a retrospective observational study. Minerva Anestesiol 2015;81:398-404.

12. Otani N, Takasato Y, Masaoka H, et al. Surgical outcome following decompressive craniectomy for poor-grade aneurysmal subarachnoid hemorrhage in patients with associated massive intracerebral or Sylvian hematomas. Cerebrovasc Dis 2008;26:6127.

13. Schirmer CM, Hoit DA, Malek AM. Decompressive hemicraniectomy for the treatment of intractable intracranial hyperten- sion after aneurysmal subarachnoid hemorrhage. Stroke 2007; 38:987-92.

14. Smith ER, Carter BS, Ogilvy CS. Proposed use of prophylactic decompressive craniectomy in poor-grade aneurysmal subarachnoid hemorrhage patients presenting with associated large sylvian hematomas. Neurosurgery 2002;51:117-24.

15. Steiner T, Juvela S, Unterberg A, et al. European Stroke Organization guidelines for the management of intracranial aneurysms and subarachnoid haemorrhage. Cerebrovasc Dis 2013;35:93112.

16. van Donkelaar CE, Bakker NA, Birks J, et al. Prediction of outcome after aneurysmal subarachnoid hemorrhage. Stroke 2019;50:837-44.

17. Alotaibi NM, Elkarim GA, Samuel N, et al. Effects of decompressive craniectomy on functional outcomes and death in poorgrade aneurysmal subarachnoid hemorrhage: a systematic review and meta-analysis. J Neurosurg 2017;127:1315-25.

18. Konczalla J, Seifert V, BeckJ, et al. Outcome after Hunt and Hess Grade V subarachnoid hemorrhage: a comparison of pre-coiling era (1980-1995) versus post-ISAT era (2005-2014). J Neurosurg 2018;128:100-10.

19. Xia ZW, Liu XM, Wang JY, et al. Coiling is not superior to clipping in patients with high-grade aneurysmal subarachnoid hemorrhage: systematic review and meta-analysis. World Neurosurg 2017;98:411-20. 